\title{
Intraoperative Navigation of an Optically Tracked Surgical Robot ${ }^{\star}$
}

\author{
Jordi Cornellà ${ }^{1,2}$, Ole Jakob Elle ${ }^{1,2}$, Wajid Ali ${ }^{1}$, and Eigil Samset ${ }^{1,2}$ \\ 1 The Interventional Centre, Rikshospitalet University Hospital, Oslo, Norway \\ ${ }^{2}$ University of Oslo, Oslo, Norway \\ jordi.cornella@rr-research.no, ole.jakob.elle@rikshospitalet.no, \\ wajid.ali@rr-research.no, eigilsa@ifi.uio.no
}

\begin{abstract}
This paper presents an adaptive control scheme for improving the performance of a surgical robot when it executes tasks autonomously. A commercial tracking system is used to correlate the robot with the preoperative plan as well as to correct the position of the robot when errors between the real and planned positions are detected. Due to the noisy signals provided by the tracking system, a Kalman filter is proposed to smooth the variations and to increase the stability of the system. The efficiency of the approach has been validated using rigid and flexible endoscopic tools, obtaining in both cases that the target points can be reached with an error less than $1 \mathrm{~mm}$. These results make the approach suitable for a range of abdominal procedures, such as autonomous repositioning of endoscopic tools or probes for percutaneous procedures.
\end{abstract}

\section{Introduction}

The aim of Computer-Integrated Surgery (CIS) systems is to make use of the advances in computing and engineering technology to overcome the limitations of traditional surgery: the combination of different preoperative image modalities together with computer image processing and modeling allows the surgeon to decide the best surgical approach; the correlation of the preoperative information with intraoperative images and robotized instruments can increase the overall accuracy of the surgical intervention; ultimately, the registration with additional images can assist in the postoperative follow-up 1 .

In spite of the growing interest for CIS systems, the range of surgical specialities where autonomous robots have been applied is still limited. Two of the most active areas in this line are neurosurgery [2] and orthopedic surgery 3], since bones and the skull are relatively easy to image and their rigidity allows an accurate and stable registration between preoperative and intraoperative images. In abdominal procedures, autonomous robots have been used in applications such as automatic positioning of the laparoscopic camera [4] or percutaneous procedures using visual servoing techniques [5. Nevertheless, due to the inherent uncertainties of most of the surgical practices, more conservative approaches like synergetic robots [6] or enhanced teleoperated systems [7] are often preferred.

\footnotetext{
* This work is funded by the E.U. contract MRTN-CT-2004-512400.
} 
An important aspect for the success of autonomous robotized applications is the correct correlation between the preoperative information and the intraoperative elements, which is frequently done by commercial optical tracking systems. Although several authors claim that optical tracking systems can measure the position of a point with a submillimetric accuracy, in a real operating room the accuracy of these systems can be strongly affected by several factors such as number of cameras, light conditions, visibility and number of targets or geometry of the system [8]. Furthermore, all these factors introduce noise in the signals provided by a tracking system, that can lead to unsafe high frequency movements or even instabilities when attempting to implement feedback control schemes with these systems. Besides the noise and accuracy of the tracking system, robots themselves can present other issues that affect the final accuracy of the system, like errors in the kinematic chain (for instance, due to non-rigid parts or gear transmissions with mechanical play), or changes in the position of the trocar point (due to breath or skin movements).

This paper presents an approach to overcome some of the inherent problems of robots and tracking systems when attempting to execute tasks autonomously. The tracking system is used not only to correlate the preoperative plan with the robot, but also to correct the position of the robot when deviations between the real and planned positions are detected. In this case, an adaptive algorithm based on a Kalman filter was developed in order to reduce the noise of the tracking signals. The approach was implemented using the commercial tracking system and robot described in our previous work [9], where the main inaccuracies of the system were also identified. In this paper, the approach is further validated using a more medically oriented setup including an abdominal phantom, and two endoscopic tools of different diameter and rigidity. The proposed algorithms can be applied in several applications, ranging from tracking the endoscopic instruments and automatic repositioning in safe or visible areas, to autonomous needle insertion in percutaneous procedures.

\section{Materials and Methods}

\subsection{Hardware Setup}

This section describes the main characteristics of the robot and tracking system used in this work.

Robotic Arm: The robotic arm is AESOP (ComputerMotion Inc., Goleta, CA, USA), a commercial voice-controlled manipulator designed for working as a camera-holder or teleoperated robot within the Zeus system. Besides the standard control modes of the robot, AESOP Development System (DS) offers a serial communication interface with a library written in $\mathrm{C}$, allowing position to be read and sent to the robot both in Cartesian and joint spaces and making it possible to develop robotic control of the manipulator.

Two different tools were designed in order to imitate the characteristics of the endoscopic instruments and percutaneous needles (Figure 1 shows an endoscopic 


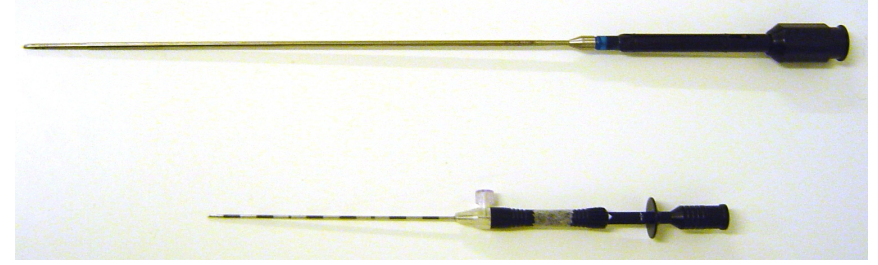

Fig. 1. Endoscopic instrument of the Zeus system and a RITA StarBurst needle for $\mathrm{RF}$ ablation of liver tumors

instrument of the Zeus system and a RITA StarBurst needle for RF ablation of liver tumors). In the first case, a $\varnothing 5 \mathrm{~mm}$ stick made of steel is used, while in the second case a RITA StarBurst needle $(\varnothing 2.2 \mathrm{~mm})$ was cut and attached to the robot.

Tracking System: The ARTtrack/DTrack (A.R.T. GmbH, Weilheim, Germany) is used as a tracking system. This is an infra-red optical tracking system composed by four cameras that can measure the position and orientation of up to 20 targets in a previously defined operating volume. The tracking system is interfaced with the robot by means of Opentracker, an open software architecture that provides a generic solution to the different tasks involved in tracking input devices [10].

\subsection{Spatial Relations}

Let $\{\mathrm{B}\}$ and $\{\mathrm{W}\}$ be the robot base and tracking system reference frames, respectively, and let ${ }^{\mathrm{B}} \boldsymbol{r}$ and ${ }^{\mathrm{w}} \boldsymbol{r}$ be the position where the endoscopic tool is attached to the robot related to $\{\mathrm{B}\}$ and $\{\mathrm{W}\}$ (see Figure 2 $\mathrm{a}$ ). The relation between ${ }^{\mathrm{w}} \boldsymbol{r}$ and ${ }^{\mathrm{B}} \boldsymbol{r}$ is given by:

$$
{ }^{\mathrm{w}} \boldsymbol{r}={ }^{\mathrm{w}} \boldsymbol{b}+{ }^{\mathrm{w}} \mathbf{R}_{\mathrm{B}}{ }^{\mathrm{B}} \boldsymbol{r}
$$

where ${ }^{\mathrm{w}} \boldsymbol{b}$ and ${ }^{\mathrm{w}} \mathbf{R}_{\mathrm{B}}$ are the position vector and the orientation matrix of $\{\mathrm{B}\}$ related to $\{W\}$, respectively.

The position of the endoscopic tool is tracked by means of a tracking target composed by five spherical retro-reflecting markers attached to its handle (Figure 21). The following elements are of special relevance when tracking and controlling the position of the endoscopic tool:

- The tip ${ }^{\mathrm{w}} \boldsymbol{e}$ : This is the point of the endoscopic tool whose position is controlled.

- The rotational center ${ }^{\mathrm{w}} \boldsymbol{c}$ : This point is the orthogonal projection of the origin of the tracking target on the axis of the endoscopic tool. As a result, ${ }^{\mathrm{w}} \boldsymbol{c}$ is not affected by the rotations of the endoscopic tool around its own axis.

- The unitary vectors ${ }^{\mathrm{w}} \hat{\boldsymbol{f}},{ }^{\mathrm{w}} \hat{\boldsymbol{g}}$ and ${ }^{\mathrm{w}} \hat{\boldsymbol{h}}$ : These three vectors define an orthogonal base with ${ }^{\mathrm{w}} \hat{\boldsymbol{f}}$ corresponding to the unitary vector of the straight line defined by the endoscopic tool. 


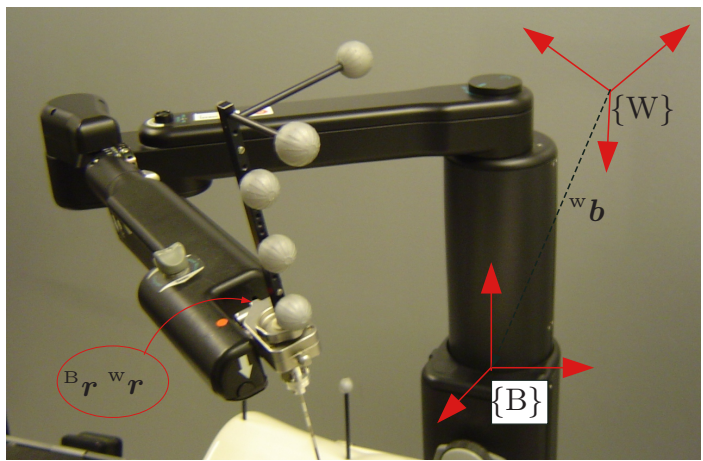

(a)

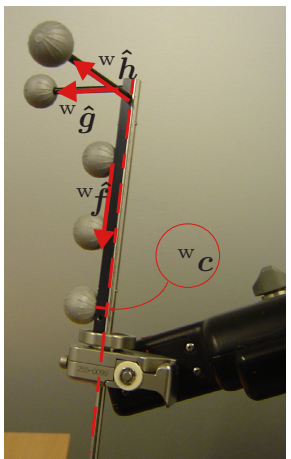

(b)

Fig. 2. (a) Relation between the robot base and tracking (world) reference frames $(\{B\}$ and $\{\mathrm{W}\}$, respectively); (b) Tracking target attached to the endoscopic tool

Let $\{\mathrm{T}\}$ be the local target reference frame. The mapping of any element from $\{\mathrm{W}\}$ to $\{\mathrm{T}\}$ or viceversa is done by the tracking system itself. Then, the above characteristic elements related to $\{\mathrm{T}\}$ are given by ${ }^{\mathrm{T}} \boldsymbol{e},{ }^{\mathrm{T}} \boldsymbol{c},{ }^{\mathrm{T}} \hat{\boldsymbol{f}},{ }^{\mathrm{T}} \hat{\boldsymbol{g}}$ and ${ }^{\mathrm{T}} \hat{\boldsymbol{h}}$, which in the ideal case would be constant. In practice, a normal distribution is obtained for each of them due to errors in the tracking system.

\subsection{Control Algorithm Structure}

The objective of the control algorithm is to make the robot follow a given preoperative plan as accurate as possible, despite the uncertainties of the system. Figure 3 shows a general overview of the proposed control algorithm. Its core is a discrete state-space feedback controller (sample time $=50 \mathrm{~ms}$ ) implemented at joint level. The performance of this controller is satisfactory, making a Cartesian position control loop unnecessary. However, when the position of the endoscopic tool needs to be controlled, several sources of uncertainty are introduced in the system and the joint controller itself is not enough. Instead of closing another control loop based on the position errors, an adaptive algorithm is proposed in order to correct those parameters that are more uncertain, namely, the position of the trocar point and the relation between the tracking system and robot.

Trocar Point: Let ${ }^{\mathrm{w}} \boldsymbol{t}^{(k)}$ be the position of the trocar point related to $\{\mathrm{W}\}$ at time $k$. Assuming that the endoscopic tool is rigid and ${ }^{\mathrm{w}} \boldsymbol{t}^{(k)} \approx^{\mathrm{w}} \boldsymbol{t}^{(k-1)}$, the rotational center ${ }^{\mathrm{w}} \boldsymbol{c}^{(k)}$ can be easily determined given a desired position ${ }^{\mathrm{w}} \boldsymbol{e}^{(k)}$ and ${ }^{\mathrm{w}} \boldsymbol{t}^{(k)}$, since these three points belong to the same line.

Although the two previous assumptions are valid, the position of the trocar can change significantly after several sample times and, therefore, an algorithm for updating its real position becomes necessary [11. In our approach, ${ }^{\mathrm{w}} \boldsymbol{t}^{(k)}$ is obtained projecting ${ }^{\mathrm{w}} \boldsymbol{t}^{(k-1)}$ on the straight line defined by ${ }^{\mathrm{w}} \boldsymbol{e}^{(k-1)}$ and ${ }^{\mathrm{w}} \boldsymbol{c}^{(k-1)}$, and then moving the resulting point a distance proportional to the position 


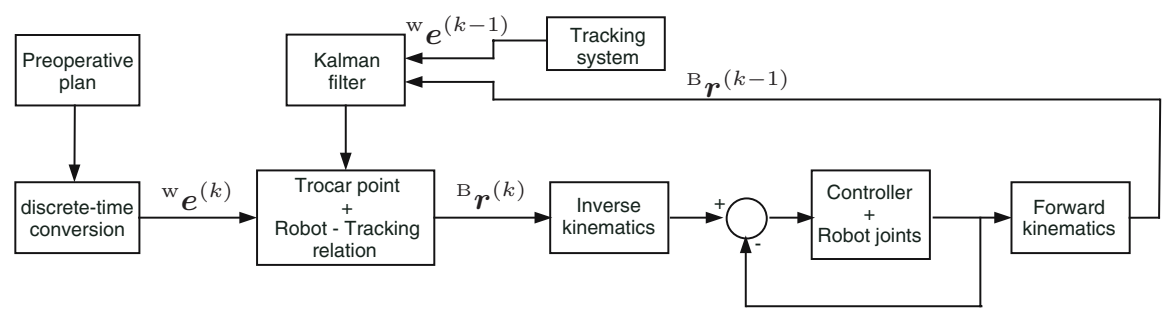

Fig. 3. Block diagram of the adaptive control algorithm

error of the endoscopic tool along its axis. This approach is simpler and more numerically stable than those based on optimization techniques.

Robot - Tracking Relation: Given the position vector ${ }^{\mathrm{w}} \boldsymbol{b}$ and orientation matrix ${ }^{\mathrm{w}} \mathbf{R}_{\mathrm{B}}$ of $\{\mathrm{B}\}$ related to $\{\mathrm{W}\}$, the relation between the robot and tracking system is determined by the following vector:

$$
\boldsymbol{v}={ }^{\mathrm{w}} \boldsymbol{r}-{ }^{\mathrm{w}} \boldsymbol{c}={ }^{\mathrm{w}} \mathbf{R}_{\mathrm{B}}{ }^{\mathrm{B}} \boldsymbol{r}+{ }^{\mathrm{w}} \boldsymbol{b}-{ }^{\mathrm{w}} \boldsymbol{c}
$$

Consider the vectors ${ }^{\mathrm{w}} \hat{\boldsymbol{f}},{ }^{\mathrm{w}} \hat{\boldsymbol{g}}$ and ${ }^{\mathrm{w}} \hat{\boldsymbol{h}}$ defined by the markers of the tracking target. The angles between these vectors and $\hat{\boldsymbol{v}}$ are given by:

$$
\cos (\alpha)=\hat{\boldsymbol{v}} \cdot{ }^{\mathrm{w}} \hat{\boldsymbol{f}} ; \quad \cos (\beta)=\hat{\boldsymbol{v}} \cdot{ }^{\mathrm{w}} \hat{\boldsymbol{g}} ; \quad \cos (\gamma)=\hat{\boldsymbol{v}} \cdot{ }^{\mathrm{w}} \hat{\boldsymbol{h}}
$$

If the attachment of the endoscopic tool to the robot was rigid, $\|\boldsymbol{v}\|, \cos (\alpha)$, $\cos (\beta)$ and $\cos (\gamma)$ would be constant values and, given ${ }^{\mathrm{w}} \boldsymbol{c}$, the corresponding robot position ${ }^{\mathrm{B}} \boldsymbol{r}$ would be easily determined solving equations (2) and (3). In practice, a normal distribution is obtained for each of these parameters due to errors in the kinematic chain.

Kalman Filter: Recomputing the above parameters every sample time can lead to high frequency movements or even instabilities, since they can change significantly from one sample to another. Then, a Kalman filter is used to update these parameters and to smooth the possible variations.

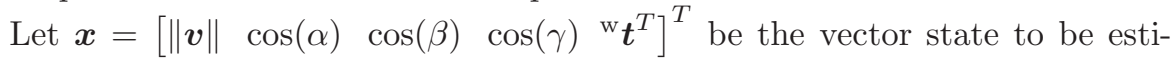
mated in a time-discrete process. All the elements of $\boldsymbol{x}$ are considered constant and independent elements, but subjected to noise. In this case, a time-discrete process can be reduced to the following equations:

$$
\begin{aligned}
& \boldsymbol{x}^{(k)}=\boldsymbol{x}^{(k-1)}+\boldsymbol{w}^{(k-1)} \\
& \boldsymbol{y}^{(k)}=\boldsymbol{x}^{(k)}+\boldsymbol{z}^{(k)}
\end{aligned}
$$

where $\boldsymbol{y}^{(k)}$ is the measured output and $\boldsymbol{w}^{(k-1)}$ and $\boldsymbol{z}^{(k)}$ are the process and measurement noise, respectively. A Kalman filter is applied independently for each element in order to smooth the variations of $\boldsymbol{x}$ (see [12 for an extended description of the Kalman filter). 


\section{Results}

This section shows the results obtained with the proposed approach. A virtual liver model (Figure 4a) was used as preoperative information in order to define the target points that the robot has to reach. This virtual model was obtained from medical images and in a real operation the target points would correspond, for instance, to possible tumors. The preoperative information was then correlated with the intraoperative robot, as it is shown in Figure 4b. An abdominal phantom was also used in order to simulate characteristics of the human skin that are difficult to model, like possible movements of the trocar point and friction forces between the skin (the phantom's neoprene belly/skin) and the needle.

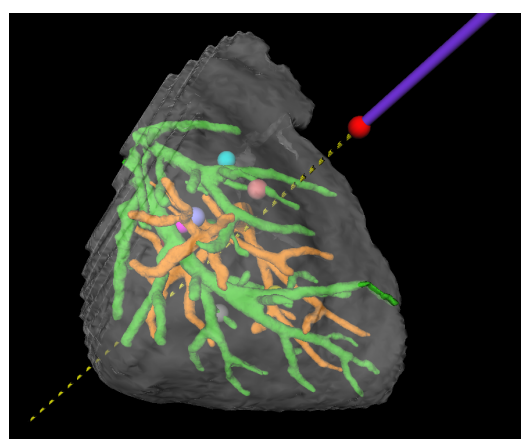

(a)

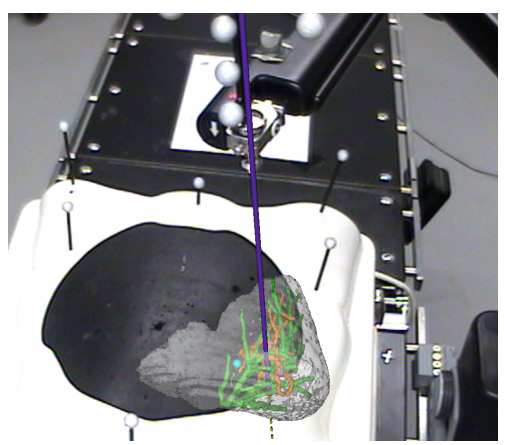

(b)

Fig. 4. (a) Virtual models of the liver and the endoscopic tool; (b) Correlation between the real robot and the virtual models

Several tests were then performed consisting of reaching the desired target points following a straight line with a typical velocity distribution (acceleration - maximum velocity - deceleration) while the error between the desired and real position was measured at each sample time. Table 1 shows the results obtained with the rigid and flexible endoscopic tools $(0.1 \mathrm{~mm}$ is considered the minimum resolution of the system). Although in both cases the final errors were similar, the maximum errors were bigger with the flexible needle, since it introduces more perturbations in the system (like bending and bigger friction forces) making it more difficult to control. The evolution of the position of the endoscopic tool, the trocar point and the internal states using the flexible needle are shown in 5 . These graphs show that, despite of the variations of the trocar and the noise

Table 1. Errors in the experimental tests (in millimeters)

\begin{tabular}{lcc}
\hline Endoscopic Tool & Maximum error & Final error \\
\hline Rigid & $2.6 \pm 0.8$ & $0.6 \pm 0.36$ \\
\hline Flexible & $5.3 \pm 2.2$ & $0.8 \pm 0.2$ \\
\hline
\end{tabular}



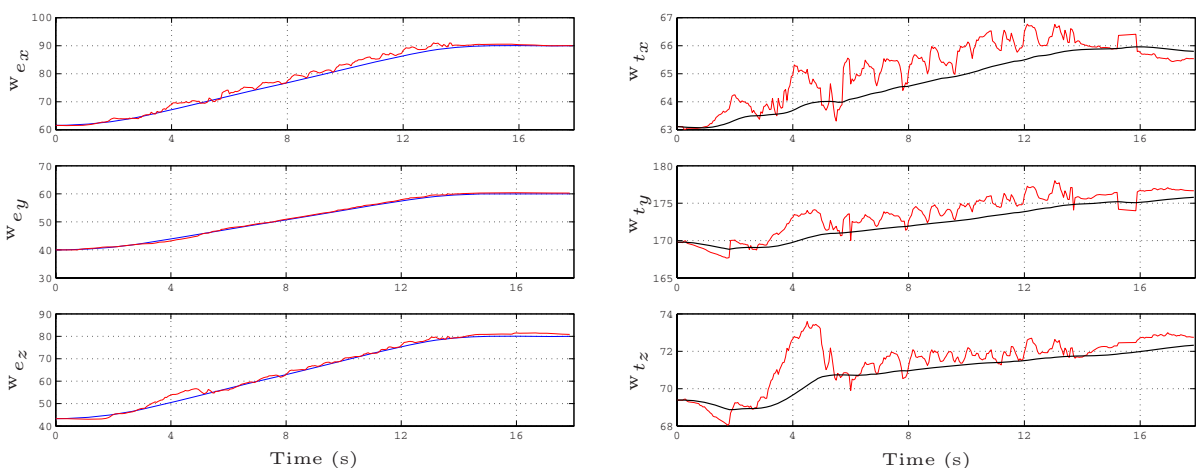

(a)

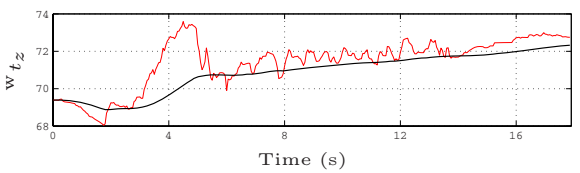

(b)

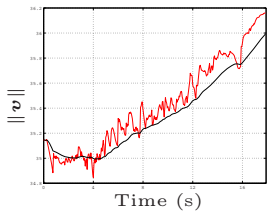

(c)

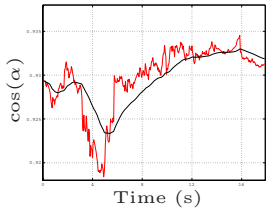

(d)

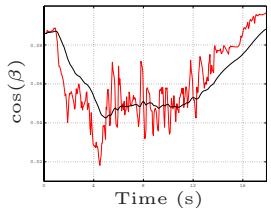

(e)

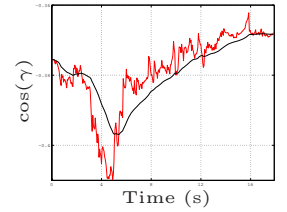

(f)

Fig. 5. Results with the flexible needle (a) Desired position (blue line) and real position (red line) of the endoscopic tool; Evolution of the vector state before and after the effect of the Kalman filter (red and black lines, respectively): (b) Trocar point; (c) $\|\boldsymbol{v}\|$; (d) $\cos (\alpha) ;(\mathrm{e}) \cos (\beta) ;(\mathrm{f}) \cos (\gamma)$.

of the parameters, the smoothing effect of the Kalman filter makes the system more stable and the trajectories can be executed with a good accuracy.

\section{Discussion}

An adaptive control scheme that improves the autonomous performance of a surgical robot has been presented in this paper. The approach has been validated using real medical equipment and an abdominal phantom for simulating the trocar point. The obtained results are very satisfactory, which encourages to test the system in more realistic setups, including for instance real organs or animal trials. However, before carrying out these tests, the absolute accuracy of the overall system (i.e., the accuracy of all the elements involved from the determination of the target point in a medical image until the robot reaches that point) must be determined, since in this paper the errors in the registration between the virtual and real target points have not been considered.

The correlation between virtual environments and the robot as it has been presented here, has additional benefits like the possibility of generating forces from the virtual environment to guide the movements of the surgeon or to avoid forbidden regions when the robot is used in a teleoperated setting. This is another interesting line to extend this work and it will be further explored in future works. 


\section{References}

1. Taylor, R.H., Stoianovici, D.: Medical robotics in computer-integrated surgery. IEEE Trans. Robot. Autom. 19(5), 765-781 (2003)

2. Karas, C., Chiocca, E.A.: Neurosurgical robotics: a review of brain and spine applications. Journal of Robotics Surgery 1, 39-43 (2007)

3. Kazanzides, P., Mittelstadt, B.D., Musits, B.L., Bargar, W.L., Zuhars, J.F., Williamson, B., Cain, P.W., Carbone, E.J.: An integrated system for cementless hip replacement. IEEE Trans. Biomed. Engin. 14(3), 307-313 (1995)

4. Krupa, A., Gangloff, J., Doignon, C., de Mathelin, M.F., Morel, G., Leroy, J., Soler, L., Marescaux, J.: Autonomous 3-d positioning of surgical instruments in robotized laparoscopic surgery using visual servoing. IEEE Trans. Robot. Autom. 19(5), 842$853(2003)$

5. Loser, M.H., Navab, N.: A new robotic system for visually controlled percutaneous interventions under ct fluoroscopy. In: Medical Image Computing and ComputerAssisted Interventions, pp. 887-896 (2000)

6. Iordachita, I., Kapoor, A., Mitchell, B., Kazanzides, P., Hager, G., Handa, J., Taylor, R.: Steady-hand manipulator for retinal surgery. In: Medical Image Computing and Computer-Assisted Interventions, pp. 66-73 (2006)

7. Li, M., Ishii, M., Taylor, R.H.: Spatial motion constraints using virtual fixtures generated by anatomy. IEEE Transactions on Robotics 23(1), 4-19 (2007)

8. Sielhorst, T., Bauer, M., Wenisch, O., Klinker, G., Navab, N.: Online estimation of the target registration error for n-ocular optical tracking systems. Medical Image Computing and Computer-Assisted Interventions, 652-659 (2007)

9. Cornellà, J., Elle, O.J., Ali, W., Samset, E.: Improving cartesian position accuracy of a telesurgical robot. In: International Symposium of Industrial Electronics (in press, 2008)

10. Reitmayr, G., Schmalstieg, D.: Opentracker - an open software architecture for reconfigurable tracking based on xml. In: Virtual Reality Conference (2001)

11. Ortmaier, T., Hirzinger, G.: Cartesian control issues for minimally invasive robot surgery. In: IEEE/RSJ Int. Conf. on Intel. Robots and Systems, pp. 565-571 (2000)

12. Welch, G., Bishop, G.: An Introduction to the Kalman Filter. Dept. of Computer Science, University of North Carolina (2004) 